\title{
Designing Reverse Supply Chain Network with Costs and Recycling Rate by Using Linear Physical Programming
}

\author{
Hiromasa Ijuin *, Yuki Kinoshita *, Tetsuo Yamada *, \\ Aya Ishigaki ${ }^{\dagger}$, Masato Inoue
}

\begin{abstract}
In recent years, economic growth and an increasing population have led to increased consumption of numerous amounts of assembly products and material resources all over the world. As the result, material shortages have become a serious global problem. To circulate materials from endof-life (EOL) assembly products, manufacturers have to design reverse supply chain networks for EOL products. The reverse supply chain includes transportation of the EOL products from collection centers to recovery and/or disposal facilities. There are costs involved in recycling, transporting the EOL products and opening facilities. In addition, the EOL product statuses differ by user situation, and the recycling rate and cost of each product and part are dependent on the statuses. To design a reverse supply chain network, a decision maker (DM) decides the transportation route, the number of products on each route, and the production volumes at each facility to minimize the total cost while maximizing the recycling rate of the whole network. However, the relationship between the recycling rate and the total cost becomes a tradeoff. Therefore, the DM has to solve these issues simultaneously. On the other hand, Linear Physical Programming (LPP) is one of the effective methods for solving multi-objective problems. It allows the DM to express desirable ranges for each criterion. One of the most significant advantages of using LPP is that the DM does not need to specify the mathematical weights for each criterion. This study designs a bi-objective reverse supply chain network to collect and recycle the EOL assembly products using LPP. First, based on our previous study, the reverse supply chain network is modeled to transport the EOL products from collection centers to recycling facilities depending on the EOL product status, which includes the possible recycling cost and rate. Next, the reverse supply chain network is formulated using LPP to minimize the total cost while maintaining the recycling rate of the whole network. Third, a case study is conducted and the results obtained by the LPP and the integer programming from our previous study are compared. Finally, the sensitivity analysis for facility cost and the effect of changing the preference ranges of objective functions are investigated.
\end{abstract}

Keywords: Reverse logistics, Environmentally Conscious Manufacturing, Multi-Criteria Decision Making, End-of-Life Product, Recycling, Disassemly

\footnotetext{
* The University of Electro-Communications, Tokyo, Japan

$\dagger$ Tokyo University of Science, Chiba, Japan

* Meiji University, Kanagawa, Japan
} 


\section{Introduction}

In recent years, global consumption of numerous amounts of assembly products and materials resources has increased due to economic growth and an increasing population. The shortage of materials is one of the most important problems around the world [1]. To develop a sustainable society, a reverse supply chain is needed for material recovery [1][2]. The reverse supply chain is a network that spans users/collection centers to recovery facilities. The End-Of-Life (EOL) products flow through the network; and become recovered materials through recycling at recovery facilities [3][4]. To increase the amount of recovered materials, the reverse supply chain is designed for the numerous EOL products that are recyclable, thereby increasing the recycling rate. On the other hand, constructing such a network is costly due to opening facilities, recycling, transportation, and final disposal of EOL products. In general, the relationship between the recycling rate and costs becomes a trade-off and it is difficult to design a reverse supply chain that satisfies both cost saving and maintains recycling rates. However, manufacturers desire lower costs but higher recycling rates for the reverse supply chain network. Therefore, the design of such a network is a bi-objective problem regarding costs and recycling rates, and it is not easy to obtain a solution that satisfies both objectives simultaneously. In addition, the goals are dependent on the DM but the DM generally struggles to decide these. However, if the goals are ranges, the burden of the DM would decrease. Furthermore, the DM needs to decide the weights for each objective. Linear physical programming (LPP) [5] [6] [7] is a well-known method used to solve multi-objective problems. It allows the DM to express their preferences as ranges for each criterion. A significant advantage of using this method is that weights allocation do not need to be specified for each criterion. The DM can therefore reflect their preferences in a more flexible and realistic manner [5].

Pati et al. [8] examined recent studies where the reverse supply chain generates profit [9] [10] [11]. Alshamsi and Diabat [9] considered production quantity and location for inspection centers and remanufacturing facilities with mixed-integer programming. In addition, other studies discussed the low-cost reverse supply chain networks of waste paper [10] and recycled sand from demolition waste [11]. Their studies focused on profit maximization of the entire reverse supply chain network. However, they have not analyzed materials recovered from inside EOL products. Ijuin et al. [12][13][14] designed the reverse supply chain network for total cost and average recycling rate using the $\varepsilon$-constraint method and analyzed recovered materials. However, the objective function of these studies is the minimization of cost, and the recycling rate is treated as an $\varepsilon$ constraint in the integer programming. Thus, the solution to satisfy the balance between the recycling rate and cost is not discussed using the LPP. To collect the recovered materials economically, this study proposes a design method of a reverse supply chain network for total cost and average recycling rate using LPP under different statuses of the EOL products.

The rest of this paper is organized as follows. Section 2 explains the model and the formulation of the reverse supply chain network for total costs and average recycling rate. Section 3 introduces LPP procedure and applies LPP to the reverse supply chain. Section 4 sets a design example for EOL products with different statuses and candidate recycling and disposal facilities to illustrate an effectiveness of the proposed method of the reverse supply chain. In addition, desired values of total costs and average recycling rate are divided to six ranges for LPP. Section 5 discusses the results of the reverse supply chain network by LPP and compares the results by an $\varepsilon$ constraint in Ijuin et al. [12]. Section 6 investigates sensitivity analysis of the fixed opening cost 
at recycling facility, and effects of changed preferences ranges by a DM on the designing reverse supply chain network. Finally, section 7 summarizes this paper and outlines future research.

\section{Modeling of reverse supply chain}

This section explains the reverse supply chain model by integer programming [15] based on [12][13][14].

\subsection{Modeling of the reverse supply chain network}

The variables and parameters of the mathematical formulation are listed as follows.

i)Indices

$i \quad:$ Objective function belonged to soft classes $(i=1, \ldots, N S C)$

$s \quad:$ Ranges $(s=2, \ldots, 5)$

$k \quad: \quad$ Status for the EOL products $(k=1, \ldots, K)$

$g \quad: \quad$ Collection centers $(g=1, \ldots, G)$

$j \quad$ : Recycling and disposal facilities $(j=1, \ldots, J)$

ii)Parameters

$K \quad: \quad$ Number of the status of EOL products

G : Number of collection centers

$J \quad: \quad$ Number of recycling and disposal facilities

$M \quad$ : Very large number (big M)

$\widetilde{w}_{i s}^{+}, \widetilde{w}_{i s}^{-} \quad: \quad$ Positive/negative deviation weight of the $s$ th range of objective $i$

$t_{i s}^{+}, t_{i s}^{-} \quad: \quad$ Positive/negative limit to the $s$ th range of objective $i$

$R C_{k j} \quad: \quad$ Recycling/Disposal cost for EOL products with the status $k$ at facility $j$

$L C_{g j} \quad: \quad$ Transportation cost from collection center $g$ to facility $j$

$F C_{j} \quad$ : The fixed opening cost at facility $j$

$R_{k j} \quad: \quad$ The possible recycling rate of status $k$ at facility $j$

$Q S_{k g} \quad: \quad$ Number of EOL products with status $k$ at collection center $g$

$B_{k j} \quad: \quad$ Available capacity of status $k$ at facility $j$

$C_{j} \quad: \quad$ Total production capacity at facility $j$

NSC : Number of soft classes 
iii)Variables

$m_{k g j} \quad: \quad$ Number of the products with status $k$ transported from collection center $g$ to facility $j$

$u_{j} \quad$ : Binary value; 1 if facility $j$ is opened, else 0

$d_{i s}^{+}, d_{i s}^{-} \quad$ : Positive/negative deviation variable from the $s$ th range limit of objective $i$

$Q D_{k j} \quad: \quad$ Number of processed EOL products with status $k$ at facility $j$

TC : Total cost of reverse supply chain

AR : Average recycling rate of the whole network

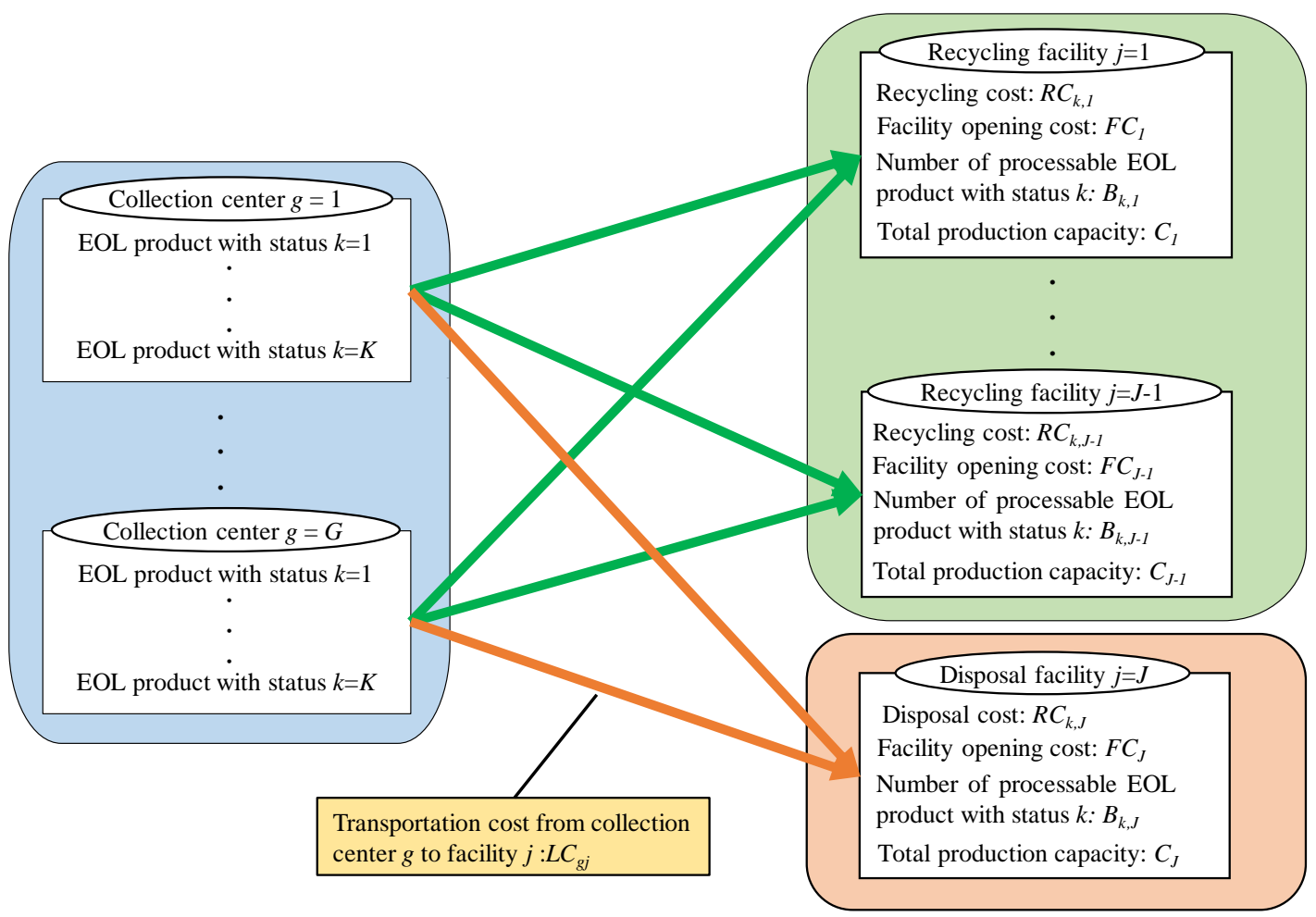

Figure 1: Model of the reverse supply chain network with EOL product status

Figure 1 shows the reverse supply chain network for EOL products based on previous research [12]. In collection center $g$, each EOL product has status $k$. The status $k$ determines possible recycling rate $R_{k j}$ and recycling cost $R C_{k j}$, and these are calculated using the recyclability evaluation method (REM) [16]. The recycling rate shows percentage of the recyclable materials out of the total weight [17]. All EOL products with status $k$ are transported from collection center $g$ to the appropriate recycling facility $j$ or disposal facility $J$ with transportation $\operatorname{cost} L C_{g j}$. When an EOL product is transported to recycling facility $j$, the recovered materials based on status $k$ are obtained. On the other hand, when the EOL product is transported to disposal facility $J$, the EOL product is disposed of. 
The total costs of the whole network $T C$ is the sum of recycling and transporting EOL products and the costs associated with opening facilities. The average recycling rate of the whole reverse supply chain $A R$ is the average recycling rate per EOL product in the revers supply chain. Therefore, when recycling facility $j$ processes the EOL products, the average recycling rate $A R$ increases based on each status $k$ of the EOL products. However, when disposal facility $J$ processes the EOL products, the average recycling rate $A R$ does not increase.

The model determines the appropriate quantities of EOL products to be transported from the collection center to the recycling and/or disposal facilities while satisfying two criteria: minimizing the total costs $T C$ and maximizing the average recycling rate of the whole network $A R$.

\subsection{Formulation of the reverse supply chain network}

This study applies LPP [5] to the design method of the reverse supply network [12] [13] [14]. The total costs $T C$ is the sum of recycling and transporting EOL products and the cost associated with opening facilities. The recycling and transportation costs are dependent on the number of transported EOL products. The facility cost is required to open the recycling or disposal facilities. Therefore, total costs $T C$ is obtained as Eq. (1).

$$
T C=\sum_{k=1}^{K} \sum_{g=1}^{G} \sum_{j=1}^{J}\left(R C_{k j}+L C_{g j}\right) m_{k g j}+\sum_{j=1}^{J} F C_{j} u_{j} \rightarrow \text { Min }
$$

On the other hand, the average recycling rate of the whole network $A R$, which is the processing rate for EOL products at the recycling facilities compared to all collected EOL products at collection centers, and is obtained as Eq. (2).

$$
A R=\frac{\sum_{k=1}^{K} \sum_{j=1}^{J-1} R_{k j} Q D_{k j}}{\sum_{g=1}^{G} \sum_{k=1}^{K} Q S_{k g}} \rightarrow \operatorname{Max}
$$

As in our previous study [12], other constraints are defined using integer programming [15]:

$$
\begin{array}{ll}
\sum_{j=1}^{J} m_{k g j}=Q S_{k g} & k=1, \ldots, K g=1, \ldots, G \\
\sum_{g=1}^{G} m_{k g j}=Q D_{k j} & k=1, \ldots, K j=1, \ldots, J \\
Q D_{k j} \leq B_{k j} & k=1, \ldots, K j=1, \ldots, J \\
\sum_{k=1}^{K} Q D_{k j} \leq C_{j} & j=1, \ldots, J \\
\sum_{k=1}^{K} Q D_{k j} \leq M u_{j} & j=1, \ldots, J \\
u_{j}=\{0,1\} & j=1, \ldots, J \\
m_{k g j}, Q D_{k j} \geq 0 & k=1, \ldots, K g=1, \ldots, G j=1, \ldots, J
\end{array}
$$




$$
m_{k g j}, Q D_{k j} \quad \text { are integers. }
$$

Equation (3) means that the total number of EOL products at the collection centers must be equal to the total number of EOL product transported. Equation (4) ensures that the total number of the transported EOL products must be equal to the total number of processed products at each recycling and/or disposal facility. Equation (5) means that the number of the EOL products with status $k$ processed in facility $j$ is equal to or less than the production capacity $B_{k j}$ based on status $k$ at facility $j$. Equation (6) means that the total amount of processed EOL products should be less than the total production capacity $C_{j}$ at facility $j$. Equation (7) ensures that the only opened facility $j$ can receive the transported EOL product. Equation (8) enforces the binary restrictions. Equations (9) and (10) enforce the non-negativity and integer restrictions.

\section{LPP Procedure}

\subsection{Overview}

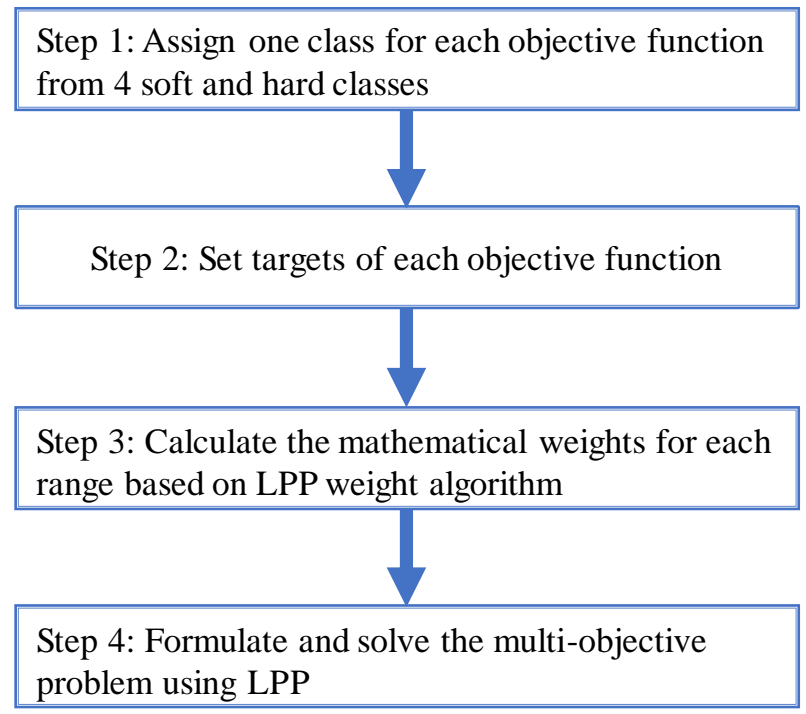

Figure 2: Procedure of solving multi-objective problem using LPP

Figure 2 shows a procedure of solving multi-objective problem using LPP [5]. Step 1 assigns one class from 4 types of hard or soft classes for each criterion. These classes refer to the sharpness of the preference. Step 2 defines the limit $t_{i l}$ through $t_{i 5}$ of the range for each criterion. Each range is set based on preferences of the DM. Step 3 generates the mathematical weights by using the LPP Weight algorithm (LPPW) [5]. Step 4 formulates and solves the reverse supply chain model using the LPP objective function. An aggregate objective function consisted of multiplied deviation variables $d_{i s}$ by weights $\widetilde{w}_{i s}$ is minimized to find a satisfied solution to reflect preferences of the DM. 


\subsection{Step 1: Assign one class for each objective function from 4 soft or hard classes}

There are 4 classes in the LPP [5]. Each class consists of two subclasses: hard and soft [5]. Both subclasses are referred to as the sharpness of the preference and are characterized by four classes. In the case of hard classes, there are four classes: must be smaller $(1 \mathrm{H})$, must be larger $(2 \mathrm{H})$, must be equal $(3 \mathrm{H})$, and must be in range $(4 \mathrm{H})[5][6]$. In addition, the hard classes are defined by only two ranges: acceptable and unacceptable.

Similar to the treatment of the hard class, there are also four types of soft classes based on DM preferences: smaller is better (1S), larger is better (2S), value is better (3S), and range is better (4S) [5][6]. In addition, each soft class has six different types of desirability ranges [5], and each range is defined by limits $t_{i s}$ of objective $i$ based on preferences of DM. Figure 3 shows soft classes functions for LPP [5]. The DM needs to specify a preference structure for each criterion to calculate weight by LPP [5].

When the DM designs the reverse supply chain network, the total costs $T C$ and average recycling rate $A R$ should be minimized and maximized, respectively. Therefore, one objective function regarding total costs $T C$ belongs to $1 \mathrm{~S}$ soft class ("smaller is better") and the function regarding average recycling rate $A R$ belongs to $2 \mathrm{~S}$ soft class ("larger is better"). The number of soft classes NSC is set as 2 .
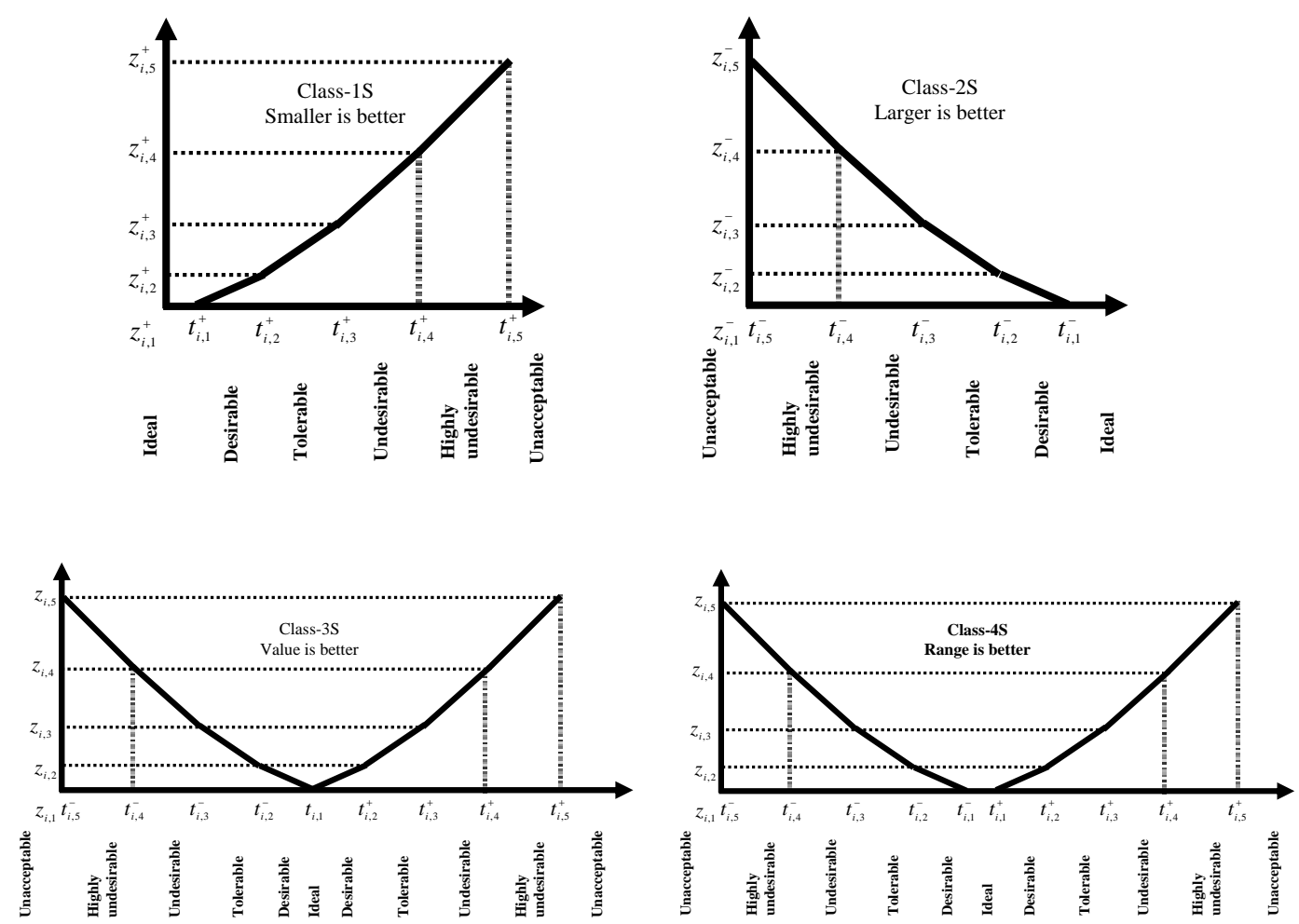

Figure 3: LPP soft class functions [5] 


\subsection{Step 2: Set targets of each objective function}

Regarding each objective function that belongs to a soft class, each range between the limit $t_{i s}$ and $t_{i s-1}(\mathrm{~s}=2,3,4,5)$ means the DM's preference level. Ranges between these limits are named "ideal," "desirable," "tolerable," "undesirable," "highly undesirable," and "unacceptable." The "ideal" is a range that is in the most desirable range among all ranges. The "desirable" and tolerable" are acceptable ranges. The "undesirable" and "highly undesirable" are ranges that, while still acceptable are not desirable. The "unacceptable" is a range of values that is not permitted for each objective.

\subsection{Step 3: Calculate the mathematical weight for each range}

Section 3.4 calculates the weight to reflect the range outlined in section 3.3. Each objective function has the deviation weights $\widetilde{w}_{i s}^{+}$or $\widetilde{w}_{i s}^{-}$for each range, which represent the weights of the $s$ th range of objective $i$ [5]. LPP has heuristic rule that is known as the one vs others criteria-rule (OVO rule) [5]. This rule means that LPP operates to improve the worst criterion, even if other criteria become worse. The quantities of the deviation weights $w^{+}{ }_{i s}$ and $w^{-}$is are not decided by the DM but are calculated based on LPP weight algorithm with OVO rule [5]. Therefore, DM is relieved from weight allocation processes to reflect his or her preferences for each criterion. The aggregate objective function is formulated to minimize the positive/negative deviation valuables for each objective function.

\subsection{Step 4: Formulate and solve the multi-objective problem using LPP}

To solve the two objectives obtained as Eqs. (1) and (2) using LPP, the aggregate objective function [5] is obtained as Eq. (11). Equation (11) means the whole objective function minimizes the sum of the deviation weights $\widetilde{w}_{i s}^{+} \widetilde{w}_{i s}^{-}$and the deviation variables $d^{+}$is $/ d_{i s}$.

$$
\sum_{i=1}^{N S C} \sum_{s=2}^{5}\left(\widetilde{w}_{i s}^{+} d_{i s}^{+}+\widetilde{w}_{i s}^{-} d_{i s}^{-}\right) \rightarrow \min
$$

The first objective of this model is related to the total costs $T C$ of the whole reverse supply chain. This objective belongs to class 1S ("smaller is better"). Therefore, the first objective is transposed as constraints (12) and (13). By minimizing sth deviation variable $d^{+}{ }_{I S}$ in Eq. (12), total costs $T C$ tries to reach ideal range $t^{+}{ }_{1 s}$. Equation (13) means that the total costs $T C$ is lower than the limit of the unacceptable $t^{+}{ }_{15}$.

$$
\begin{aligned}
& T C-d_{1, s}^{+} \leq t_{1, s-1}^{+} \quad s=2, \ldots, 5 \\
& T C \leq t_{1,5}^{+}
\end{aligned}
$$


The second objective function is related to the average recycling rate of the whole reverse supply chain network $A R$. This objective belongs to class $2 \mathrm{~S}$ ("larger is better"). Therefore, the second objective is obtained as (14) and (15). Similar to (12), the average recycling rate $A R$ tries to reach ideal range by minimizing sth variable $d^{+}{ }_{2 s}$. Equation (15) means that the average recycling rate $A R$ is higher than the limit of the unacceptable $t^{+}{ }_{25}$. Equation (16) enforces the non-negativity restrictions.

$$
\begin{array}{ll}
A R+d_{2, s}^{-} \geq t_{2, s-1}^{-} & s=2, \ldots, 5 \\
A R \geq t_{2,5}^{-} & \\
d_{i s}^{+}, d_{i s}^{-} \geq 0 & i=1, \ldots, N S C, s=2, \ldots, 5
\end{array}
$$

\section{Example problem}

\subsection{EOL product example}

To validate the proposed design with LPP, this study uses the same example from previous study [12] on the product and the reverse supply chain network. The vacuum cleaner used as the product example has 23 parts and four disassembly scenarios based on Igarashi et al. [18]. Table 1 lists the statuses of EOL products in the case of vacuum cleaner obtained from 4 disassembly scenarios [18]. The required number of disassembly stations depends on the EOL product status as shown in table 1. Additionally, the recycling rate and cost of the EOL product depend on the 4 disassembly scenarios. According to Akahori et al. [17], the recycling cost includes disassembly, processing, and disposal costs. The recycling cost is calculated based on disassembly time by the REM developed by Hitachi Ltd. [16].

Table 1: Product status based on the Igarashi disassembly scenario: case of a vacuum

$$
\text { cleaner [18] }
$$

\begin{tabular}{c|c|c}
\hline $\begin{array}{c}\text { EOL product } \\
\text { status: } \boldsymbol{k}\end{array}$ & Scenario & $\begin{array}{c}\text { Required number of } \\
\text { stations for recycling }\end{array}$ \\
\hline \hline 1 & All parts disas sembled & 8 \\
\hline 2 & Recycling rate maximum & 8 \\
\hline 3 & Recycling cost minimum & 1 \\
\hline 4 & Recycling rate and cost coexistence & 3 \\
\hline
\end{tabular}




\subsection{Recycling and disposal facilities example}

This subsection prepares an example of the reverse supply chain network for locations (distances), production capacities, and the number of recycling work stations at recycling or disposal facilities, and explains the processable statuses of EOL product for each recycling facility. As listed in table 1, there are different statues of the EOL products. With respect to the recycling and disposal facilities, there are 3 recycling facilities with different number of stations and 1 disposal facility in the reverse supply chain. Table 2 shows the number of stations for recycling, production capacity for each recycling or disposal facility, and fixed opening cost. The processable statuses of the EOL products at each recycling facility are determined based on the number of stations as shown in tables 1 and 2. For example, when a EOL product with status 1 has the "all parts disassembled" scenario, only recycling facility 1 with 8 disassembly stations is available to be recycled the EOL product with status 1 .

With respect to each recycling rate and cost of the different EOL product statuses, table 3 shows recycling rate, cost and number of processable production capacity for each status. As each recycling facility has different processable statues of the EOL products, the recycling rate at recycling facility $j R_{k j}$ depends on the status of EOL product and recycling facilities. Similar to the recycling rate $R_{k j}$, the recycling cost $R C_{k j}$ depends on the status and recycling facilities. For example, since the status 1 "all the disassembled parts" can be recycled at only recycling facility 1 , the recycling cost of status 1 at facility $1 R C_{11}$ is 402.17 while the recycling rate of status 1 at facility $1 R_{11}$ is $95.48 \%$.

The maximal production capacity of EOL product at facility $j$ in table 2 is represented as the total number of production capacity $C_{j}$. On the other hand, production capacity for each status $k$ in table 3, $B_{k j}$, means the processible number of EOL products with status $k$ at the recycling facility $j$. Thus, the sum of EOL products with status $k\left(\sum_{k=1}^{K} B_{k j}\right)$ is equal to or less than total production capacity $C_{j}$ for each recycling facility. The transportation cost from collection center to recycling/disposal facility are set based on the distance between them as shown in table 4.

Table 2: Number of stations for recycling, production capacity, and fixed opening cost for each facility: case of a vacuum cleaner [12]

\begin{tabular}{cccc}
\hline $\begin{array}{c}\text { Recycling/Disposal } \\
\text { facility: } \boldsymbol{j}\end{array}$ & $\begin{array}{c}\text { Number of } \\
\text { stations for } \\
\text { recycling }\end{array}$ & $\begin{array}{c}\text { Number of } \\
\text { production capacity: } \\
\boldsymbol{C}_{\boldsymbol{j}} \text { [units] }\end{array}$ & $\begin{array}{c}\text { Fixed opening cost } \\
\text { at facility } \boldsymbol{j}: \\
\boldsymbol{F} \boldsymbol{C}_{\boldsymbol{j}}\end{array}$ \\
\hline \hline Recycling facility $(j=1)$ & 8 & 12,000 & 800,000 \\
Recycling facility $(j=2)$ & 3 & 12,000 & 400,000 \\
Recycling facility $(j=3)$ & 1 & 12,000 & 100,000 \\
Disposal facility $(j=4)$ & - & 100,000 & 0 \\
\hline
\end{tabular}


Table 3: Recycling rate, cost and number of processable production capacity for each status

\begin{tabular}{|c|c|c|c|c|c|c|c|c|c|c|c|c|}
\hline \multirow{2}{*}{$\begin{array}{c}\begin{array}{c}\text { Recycling/Disposal } \\
\text { facility: } j\end{array} \\
\text { EOL product status: } k\end{array}$} & \multicolumn{3}{|c|}{ Recycling facility $(j=1)$} & \multicolumn{3}{|c|}{ Recycling facility $(j=2)$} & \multicolumn{3}{|c|}{ Recycling facility $(j=3)$} & \multicolumn{3}{|c|}{ Disposal facility $(j=4)$} \\
\hline & $\begin{array}{c}\text { Recycling } \\
\text { cost } \\
R C_{k j}\end{array}$ & $\begin{array}{c}\text { Recycling } \\
\text { rate } \\
R_{k j}[\%]\end{array}$ & $\begin{array}{c}\text { Number of } \\
\text { processable EOL } \\
\text { product with status } k \\
\text { at facility } j: B_{k j}\end{array}$ & $\begin{array}{c}\text { Recycling } \\
\text { cost } \\
R C_{k j}\end{array}$ & $\begin{array}{c}\text { Recycling } \\
\text { rate } \\
R_{k j}[\%]\end{array}$ & $\begin{array}{c}\text { Number of } \\
\text { processable EOL } \\
\text { product with status } k \\
\text { at facility } j: B_{k j}\end{array}$ & $\begin{array}{c}\text { Recycling } \\
\text { cost } \\
R C_{k j}\end{array}$ & $\begin{array}{c}\text { Recycling } \\
\text { rate } \\
\boldsymbol{R}_{k j}[\%]\end{array}$ & $\begin{array}{c}\text { Number of } \\
\text { processable EOL } \\
\text { product with status } k \\
\text { at facility } j: B_{k j}\end{array}$ & $\begin{array}{c}\text { Recycling } \\
\text { cost } \\
R C_{k j}\end{array}$ & $\begin{array}{c}\text { Recycling } \\
\text { rate } \\
\boldsymbol{R}_{k j}[\%]\end{array}$ & $\begin{array}{c}\text { Number of } \\
\text { processable EOL } \\
\text { product with status } k \\
\text { at facility } j: B_{k j}\end{array}$ \\
\hline 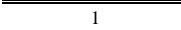 & 402.17 & "95.48 & 12,000 & 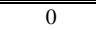 & 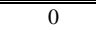 & 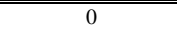 & 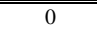 & 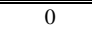 & 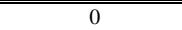 & $\overline{00}$ & 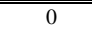 & 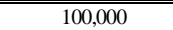 \\
\hline 2 & 383.76 & 95.48 & 12,000 & 0 & 0 & 0 & 0 & 0 & 0 & 0 & 0 & 100,000 \\
\hline 3 & 36.51 & 13.10 & 12,000 & 36.51 & 13.10 & 12,000 & 36.51 & 13.10 & 12,000 & 0 & 0 & 100,000 \\
\hline 4 & 152.65 & 64.02 & 12,000 & 152.65 & 64.02 & 12,000 & 0 & 0 & 0 & 0 & 0 & 100,000 \\
\hline
\end{tabular}

Table 4: Transportation cost from collection center to recycling/disposal facility: case of a vacuum cleaner [12]

\begin{tabular}{ccc}
\hline $\begin{array}{c}\text { Recycling/Disposal } \\
\text { facility } \boldsymbol{j}\end{array}$ & \multicolumn{2}{c}{ Collection center $\boldsymbol{i}$} \\
& $\boldsymbol{i}=\mathbf{1}$ & $\boldsymbol{i}=\mathbf{2}$ \\
\hline \hline Recy cling facility $(j=1)$ & 351.0 & 433.0 \\
Recy cling facility $(j=2)$ & 47.5 & 189.5 \\
Recy cling facility $(j=3)$ & 204.5 & 9.5 \\
Disposal facility $(j=4)$ & 73.0 & 189.0 \\
\hline
\end{tabular}




\subsection{Set LPP preference}

In the soft classes in LPP [5][6][7], a DM needs to set five limits $t_{i s}^{+}, t_{i s}^{-}$based on his/her preferences to define six different desirability ranges. Thus, this study provides the range of each objective function as listed in Table 5. Table 5 lists preferences with the limit $t_{i s}^{+}, t_{i s}^{-}$, which represents the total costs $T C$ or the average recycling rate $A R$ of the whole network.

Table 5: Preference table of LPP

\begin{tabular}{c|c|c}
\hline Preference level & Total costs: $\boldsymbol{T C}$ & Average recycling rate: $\boldsymbol{A R}$ \\
\hline Ideal & $\leq 3,000,000$ & $\geq 60 \%$ \\
\hline Desirable & $(3,000,000,5,500,000]$ & {$[47.5 \%, 60 \%)$} \\
\hline Tolerable & $(5,500,000,8,000,000]$ & {$[35 \%, 47.5 \%)$} \\
\hline Undesirable & $(8,000,000,10,500,000]$ & {$[22.5 \%, 35 \%)$} \\
\hline Highly Undesirable & $(10,500,000,12,000,000]$ & {$[10 \%, 22.5 \%)$} \\
\hline Unacceptable & $>12,000,000$ & $<10 \%$ \\
\hline
\end{tabular}

\section{The result of reverse supply chain network by LPP}

\subsection{LPP weights parameters}

Table 6 lists the mathematical weights $w^{+}$is and $w^{-}$is calculated by LPP weight algorithm. The calculated mathematical weights reflect to the preferences of the DM in table 5. The objective function of the total costs TC belongs to class $1 \mathrm{~S}$ ("smaller is better"). In contrast to the total costs $T C$, another objective function of the average recycling rate $A R$ belongs to the class $2 \mathrm{~S}$ ("larger is better"). By using the weight as shown in table 6, LPP can find one solution to reflect the preferences of DM.

Table 6: Mathematical weights calculated by LPP weight algorithm for the total costs $T C$ and average recycling rate $A R$

\begin{tabular}{lllll}
\hline \multicolumn{1}{c}{ Objective function } & \multicolumn{4}{c}{ Calculated LPP weight } \\
\hline \hline Total costs $(T C)$ & $\widetilde{w}_{1,2}^{+}=0.0160$ & $\widetilde{w}_{1,3}^{+}=0.0150$ & $\widetilde{w}_{1,4}^{+}=0.0292$ & $\widetilde{w}_{1,5}^{+}=0.1345$ \\
Averagre recycling rate $(A R)$ & $\widetilde{w}_{2.2}^{-}=3077$ & $\widetilde{w}_{2 .}^{-}=3390$ & $\widetilde{w}_{2,}^{-}=5114$ & $\widetilde{w}_{2.5}^{-}=12758$ \\
\hline
\end{tabular}

\subsection{Total cost and recycling rate}

Table 7 shows the results of value and aspiration level of each objective. The aspiration levels mean which preference levels correspond to the obtained value of the objective using LPP. From Table 7, the total costs $T C$ becomes 7,999,960, which corresponds to the "tolerable" range. In addition, the average recycling rate $A R$ becomes $38.77 \%$, which corresponds to the "tolerable" range. However, the total costs $T C$ is very close to an undesirable level $(>8,000,000)$. Therefore, it is observed that the higher priority is given to maximizing the recycling rate $A R$ than to minimizing the total costs $T C$. One of the reasons for this is that as the average recycling rate $A R$ becomes higher, the average recycling rate per unit of the total $\operatorname{cost}(=$ the average recycling rate/total cost) is improved. If both objective functions are at 
the same level, it is considered that the higher priority is given to maximizing the recycling rate rather than to minimizing the total costs $T C$ in the numerical experiments.

Table 7: Results of value and aspiration level of each objective

\begin{tabular}{ccc}
\hline Objective function & Value & Aspiration level \\
\hline \hline Total costs $(T C)$ & $7,999,960$ & Tolerable \\
\hline Averagre recycling rate $(A R)$ & $38.77 \%$ & Tolerable \\
\hline
\end{tabular}

Table 8: Results of the number of transported EOL products from the collection center to the recycling/disposal facility: case of the vacuum cleaner

\begin{tabular}{ccc|c|c|c}
\hline \multirow{2}{*}{$\begin{array}{c}\text { Collection } \\
\text { center } \boldsymbol{i} \boldsymbol{i}\end{array}$} & $\begin{array}{r}\text { EOL } \\
\text { product } \\
\text { status: } \boldsymbol{k}\end{array}$ & \multicolumn{5}{c}{ Recycling/Disposal facility: $\boldsymbol{j}$} \\
\cline { 3 - 6 } & & $\begin{array}{c}\text { Recycling } \\
\text { facility }(j=1)\end{array}$ & $\begin{array}{c}\text { Recycling } \\
\text { facility }(j=2)\end{array}$ & $\begin{array}{c}\text { Recycling } \\
\text { facility }(j=3)\end{array}$ & $\begin{array}{c}\text { Disposal facility } \\
(j=4)\end{array}$ \\
\hline \hline & 1 & 0 & 0 & 0 & 3,000 \\
1 & 2 & 0 & 0 & 0 & 3,000 \\
& 3 & 0 & 3,000 & 0 & 0 \\
& 4 & 0 & 3,000 & 0 & 0 \\
\hline \multirow{2}{*}{2} & 1 & 1,900 & 0 & 0 & 1,100 \\
& 2 & 3,000 & 0 & 0 & 0 \\
& 3 & 0 & 0 & 3,000 & 0 \\
& 4 & 0 & 3,000 & 0 & 0 \\
\hline
\end{tabular}

Table 8 shows the results of the number of transported EOL products from the collection center to the recycling/disposal facility in the case of a vacuum cleaner. For example, it was found that 3,000 units of the EOL products with status 1 at the collection center 1 was transported to the disposal facility. Additionally, the recycling facility 2 received 9,000 units of the EOL products from both collection centers 1 and 2. It is observed that all recycling facilities 1,2 , and 3 were opened, even though the additional fixed opening costs was required for each recycling facility. Moreover, the recycling facility 2 was still available to receive more 3,000 units of the EOL products since the total capacity of the recycling center 2 was 12,000 units. However, all EOL products with status 3 at collection center 2 were transported to the recycling facility 3 . Then, the additional fixed opening cost for the recycling facility 3 such as 100,000 was required. The reason why the recycling facility 3 was opened was that there were the difference of transporting costs from the collection center 2 to recycling facilities 2 and 3 as shown in table 4 . The transport cost between the collection center 2 and the recycling facility 3 is by $95 \%$ lower than one between the collection center 2 and the recycling facility 2 . Therefore, by transporting all EOL product with the status 3 at the collection center 2 to the recycling facility 3 instead of recycling facility 2, the total costs $T C$ in the reverse supply chain could be saved by $75 \%$. 


\subsection{LPP vs $\varepsilon$ constraint method}

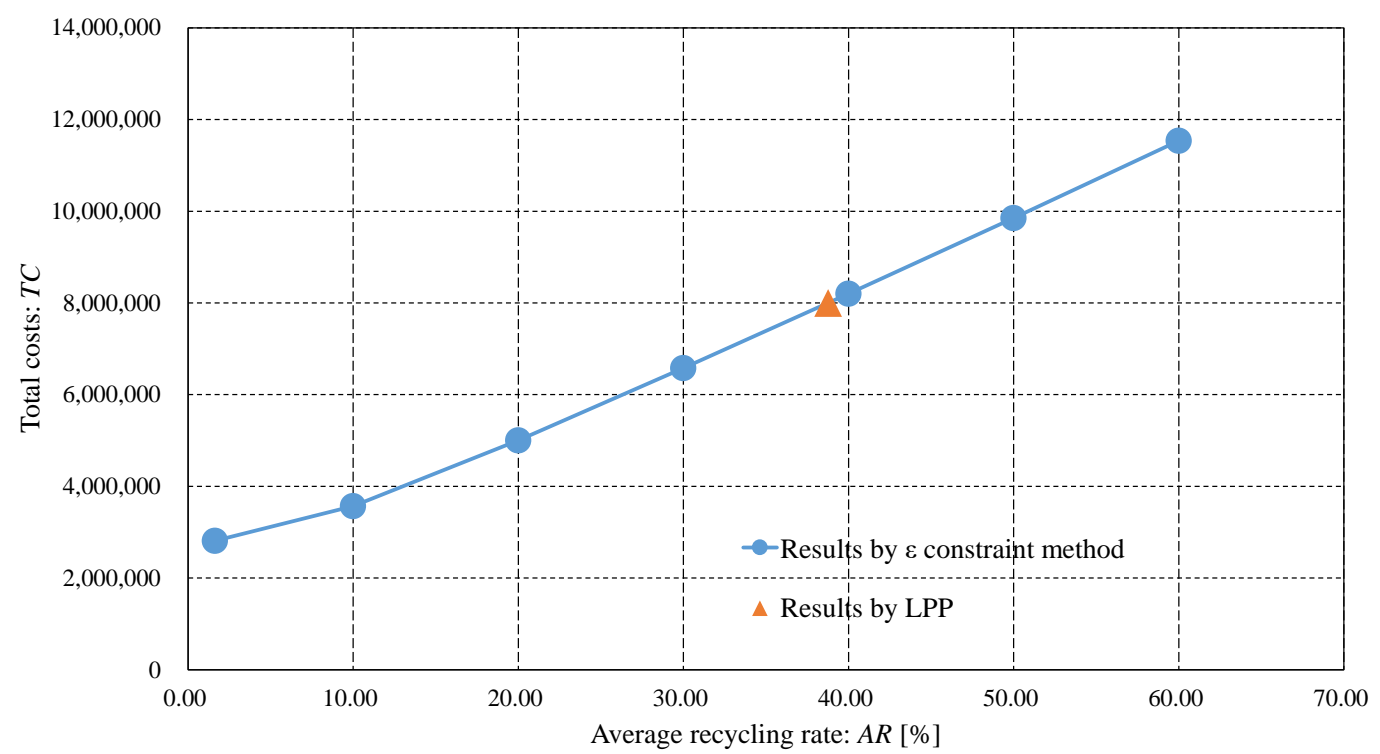

Figure 4: Behaviors of total costs $T C$ and average recycling rate $A R$ using LPP with $\varepsilon$ constraint method

This section compares the results by LPP and by $\varepsilon$ constraint method [12] to validate the total costs $T C$ and average recycling rate $A R$ by LPP. The $\varepsilon$-constraint method prioritizes a primary objective while expressing other objectives as constraints to solve multi-objective problems [2]. By changing values of constraints related to non-prioritized objectives, the Pareto front is approximated [2]. Ijuin et al. [12] applied the $\varepsilon$-constraint method to designing the reverse supply chain network to obtain Pareto optimal solutions for the total costs $T C$ and average of recycling rate $A R$.

Figure 4 shows the behaviors of the total costs $T C$ and average recycling rate $A R$ solved using LPP and $\varepsilon$ constraints method [12]. The vertical axis represents the total costs $T C$, while the horizontal axis means the average recycling rate $A R$. The circles connected by the blue line in Fig. 4 represent the results of $\varepsilon$ constraint method in [12], while the orange triangle indicates the result of LPP. Pareto frontier for the total costs $T C$ vs the average recycling rate $A R$ of the whole network $A R$ can be observed in Fig. 4. The resulted value by LPP was located on the blue line between solutions with $30 \%$ and $40 \%$ average recycling rates $A R$. Thus, LPP could obtain one solution to satisfy desirable levels for the total costs $T C$ and average recycling rate simultaneously with lower runs than that by $\varepsilon$ constraint method. 


\section{Effect by recycling facility cost and different ranges of objec- tive functions}

\subsection{Fluctuation of facility cost at recycling facility}

This study has investigated the reverse supply chain to increase the recycling rate with the cost opening facilities, transportation and recycling EOL products. Regarding decision making for a reverse supply chain configuration, the opening recycling facility cost is one of the most important factors. This is because if a facility is built at once, the manufacturers cannot easily change the location. Therefore, the opening facility costs have significant impacts in terms of designing the reverse supply chain network. On the other hand, the preference ranges set by a DM are also an important factor since mathematical weights to construct the reverse supply chain with LPP are calculated based on the preference ranges of the DM. For example, if the DM wants to decrease costs further, they may decide to introduce an extreme lower cost range that maintains a recycling rate. Thus, section 6.1 conducts sensitivity analysis of opening cost for recycling facilities to examine the effect of opening recycling facility costs on the reverse supply chain configuration. Section 6.2 investigates the total costs $T C$ and average recycling rate $A R$ under different preference ranges.

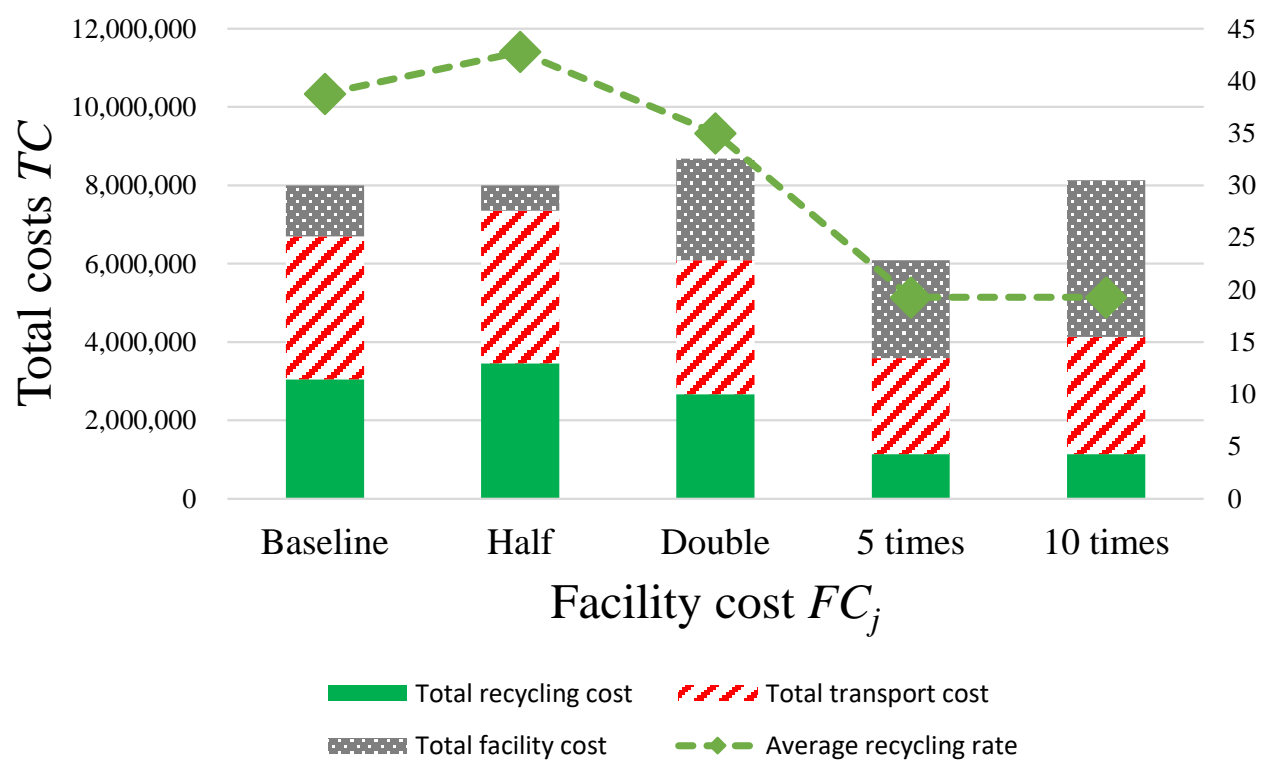

Figure 5: Behaviors of total costs $T C$ and average recycling rate $A R$ for recycling facility cost 
In the sensitivity analysis of the opening cost for recycling facilities, the opening costs at recycling facility are assumed to set as half, double, five, and 10 times higher than the that of initial input data (see table 2) in the numerical experiment at section 5. Figure 5 shows total costs $T C$ breakdown and average recycling rate $A R$ of the sensitivity analysis of the opening cost at recycling facility. The baseline refers the results conducted in the section 5 using the initial input data. At Fig. 5, green bar represents to total recycling cost, red bar indicates total transportation costs, and gray bar shows total facility cost. Since the opening cost for the disposal facility is assumed to be 0 , the total disposal cost is not appeared in any scenarios in Fig 5. The green line represents the average recycling rate $A R$.

Compared the case of half facility cost with the baseline, the average recycling rate $A R$ in the case of half facility cost was $4 \%$ higher than that of the baseline, even though the total costs $T C$ was the almost same within $1 \%$. Therefore, the half of facility costs did not contribute reduction of the total cost $T C$; however, it could increase the average recycling rate $A R$.

In the cases of double and 5 times higher facility costs, the total opening cost at the recycling facility increased 2 and 1.9 times respectively, and the average recycling rate $A R$ decreased by $3.8 \%$ and $19.5 \%$, respectively. In the case of 10 times higher facility cost, the total costs $T C$ increased by $1.6 \%$ from the baseline. Moreover, the average recycling rate $A R$ in the case of 10 times higher facility cost was the same as that in the case of 5 times higher facility one. From the Fig. 5, it was observed that the total costs $T C$ increased within $10 \%$ from that of baseline among half, double and 10 times higher facility cost. 


\subsection{Fluctuation of facility cost at recycling facility}

Sections 6.2 investigates the effect of different ranges on the reverse supply chain configuration for the average recycling rate $A R$ and total costs $T C$. It assumes the following three scenarios: Cost oriented Recycling oriented, and Recycling and cost coexistence. Table 9 presents the changed ranges of average recycling rate $A R$ and the total costs $T C$. Based on the changed total costs $T C$ and/or average recycling rate $A R$ ranges, LPP weight were calculated for each scenario to design the reverse supply chain network using LPP. In the baseline, the range is obtained from Table 5.

Table 9. Changed ranges of total costs $T C$ and average recycling rate $A R$

\begin{tabular}{|c|c|c|c|}
\hline $\begin{array}{l}\text { Scenarios for } \\
\text { changing ranges }\end{array}$ & Preference level & Total cost: $T C$ & Average recycling rate: $A R$ \\
\hline \multirow{6}{*}{ Cost-oriented } & Ideal & $\leq 3,000,000$ & $\geq 60 \%$ \\
\hline & Desirable & $(3,000,000,3,500,000]$ & {$[47.5 \%, 60 \%)$} \\
\hline & Tolerable & $(3,500,000,5,000,000)]$ & {$[35 \%, 47.5 \%)$} \\
\hline & Undesirable & $(5,000,000,7,500,000]$ & {$[22.5 \%, 35 \%)$} \\
\hline & Highly Undesirable & $(7,500,000,12,000,000]$ & {$[10 \%, 22.5 \%)$} \\
\hline & Unacceptable & $>12,000,000$ & $<10 \%$ \\
\hline \multirow{6}{*}{$\begin{array}{l}\text { Recycling- } \\
\text { oriented }\end{array}$} & Ideal & $\leq 3,000,000$ & $\geq 65 \%$ \\
\hline & Desirable & $(3,000,000,5,500,000]$ & {$[60 \%, 65 \%)$} \\
\hline & Tolerable & $(5,500,000,8,000,000]$ & {$[51 \%, 60 \%)$} \\
\hline & Undesirable & $(8,000,000,10,500,000]$ & {$[38 \%, 51 \%)$} \\
\hline & Highly Undesirable & $(10,500,000,12,000,000]$ & {$[20 \%, 38 \%)$} \\
\hline & Unacceptable & $>12,000,000$ & $>20 \%$ \\
\hline \multirow{6}{*}{$\begin{array}{l}\text { Recycling and } \\
\text { cost coexistence }\end{array}$} & Ideal & $\leq 3,000,000$ & $\geq 65 \%$ \\
\hline & Desirable & $(3,000,000,3,500,000]$ & {$[60 \%, 65 \%)$} \\
\hline & Tolerable & $(3,500,000,5,000,000)]$ & {$[51 \%, 60 \%)$} \\
\hline & Undesirable & $(5,000,000,7,500,000]$ & {$[38 \%, 51 \%)$} \\
\hline & Highly Undesirable & $(7,500,000,12,000,000]$ & {$[20 \%, 38 \%)$} \\
\hline & Unacceptable & $>12,000,000$ & $>20 \%$ \\
\hline
\end{tabular}

Figure 6 shows the average recycling rate $A R$ and total costs $T C$ among scenarios of costoriented, recycling-oriented, recycling and cost coexistence and baseline. The blue circle represents the baseline. The orange triangle, red diamond, and green square represent scenarios of Cost-oriented, Recycling-oriented, and Recycling and cost coexistence, respectively. In all scenarios, the total costs $T C$ is obtained between 7,000,000 and 10,500,000, and the average recycling rate $A R$ remains over $35 \%$. The highest average recycling rate $A R$ among the 4 scenarios was the recycling-oriented one as shown in Fig. 6. Additionally, the lowest total costs $T C$ among 4 scenarios was in the cost-oriented one. However, the solutions with higher average recycling rates $A R$ such as $60 \%$ and lower total costs $T C$ under 4,000,000 obtained by $\varepsilon$ constraint method as shown in Fig. 4 were not found in the changed preferences ranges by LPP. Therefore, even though LPP would not find total costs $T C$ minimum and average recycling rate $A R$ maximum solutions, LPP can seek solutions to satisfy desirability for the total costs $T C$ and average recycling rate $A R$ at once run. 


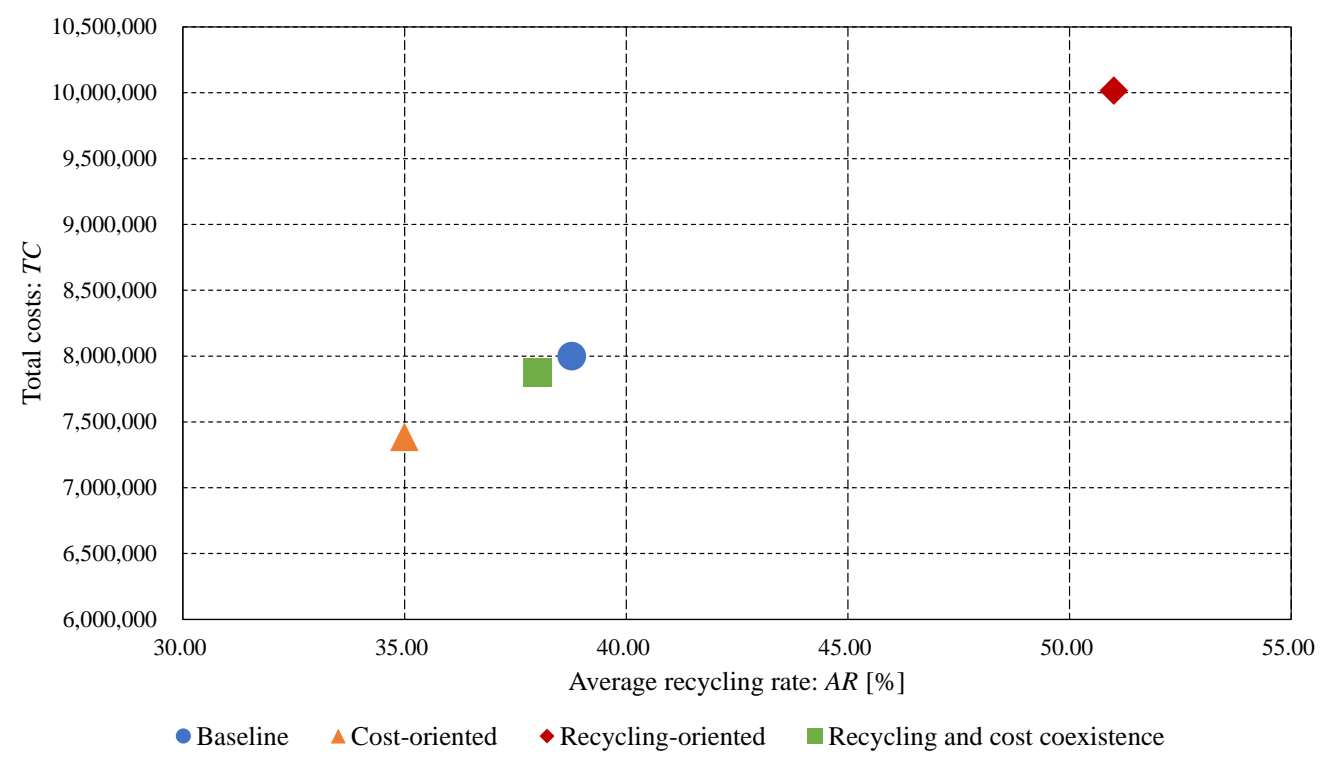

Figure 6: Total costs $T C$ and average recycling rate $A R$ with changed preference ranges of a DM.

\section{Conclusions}

This study modeled and formulated the reverse supply chain network with LPP in terms of total costs $T C$ and average recycling rate $A R$. The result of LPP harmonized both the objective functions. The main conclusions of this paper are as follows:

- Using the LPP, the greater priority was given to maximize the recycling rate than to minimize the total costs $T C$ in the numerical experiments.

- The case was found in the numerical experiments that an additional recycling facility was opened with the fixed opening cost to save the total costs $T C$ in the reverse supply chain, even though other recycling facilities had still capacity to receive the EOL products.

- By comparing LPP with $\varepsilon$ constraint method, LPP finds a satisfied solution with the total costs $T C$ and average recycling rate $A R$ based on the preferences of the DM with lower number of experimental trials.

- When the fixed opening cost at recycling facility cost was lower, the average recycling rate $A R$ was improved. On the other hand, when the fixed opening costs at recycling facility cost are higher, fewer facilities were opened then the average recycling rate $A R$ became lower.

- In contrast to $\varepsilon$ constraint method, LPP would not find total costs $T C$ minimum and average recycling rate $A R$ maximum solutions, even though the preference ranges of the DM was set as recycling or cost-oriented scenarios. However, LPP could seek solutions to satisfy desirability for the total costs $T C$ and average recycling rate $A R$ at once run.

Future work should consider increasing the number of objective functions such as recovered materials and integrating a mixed collection for multiple types of products. 


\section{Acknowledgments}

The authors would like to thank Mr. Seiichi Fujita and Hitachi Ltd. for providing the REM software. This research was partially supported by the Japan Society for the Promotion of Science (JSPS), KAKENHI, Grant-in-Aid Research (A), JP18H03824, from 2018 to 2020.

\section{References}

[1] H. F. Wang and S.M. Gupta, Green Supply Chain Management: Product Life Cycle Approach, McGraw-Hill, New York, NY, USA, 2011.

[2] M. Eskandarpour, P. Dejax, J. Miemczyk, O. Péton, "Sustainable supply chain network design: an optimization-oriented review," Omega, vol.54, pp.11-32, 2015.

[3] K. Govindan, H. Soleimani, D. Kannan, "Reverse logistics and closed-loop supply chain: a comprehensive review to explore the future," European Journal of Operational Research, vol.240, no.3, pp.603-626, 2015.

[4] D. F. Blumberg, Introduction to Management of Reverse Logistics and Closed Loop Supply Chain Processes, Boca Raton, FL, USA, CRC Press, 2005.

[5] A. Messac, S. M. Gupta, and B. Akbulut, "Linear physical programming: a new approach to multiple objective optimization," Transactions on Operational Research, vol.8, no.2, pp.3959, 1996.

[6] M. A. Ilgin, S. M. Gupta, "Physical programming: a review of the state of the art," Studies in Informatics and Control, vol.21, no.4, pp.349-366, 2012.

[7] O. Ondemir, S. M. Gupta, "A multi-criteria decision making model for advanced repair-toorder and disassembly-to-order system," European Journal of Operational Research, vol.233, no.2, pp.408-419, 2014.

[8] R.K. Pati, R. Jans, R.K. Tyagi, “Green logistics network design: a critical review,” Production and Operations Management Society 24th Annual Conference, 6 pages, Denver, CO, USA, May, 2013.

[9] A. Alshamsi, A. Diabat, “A reverse logistics network design,” Journal of Manufacturing Systems, vol.37, no.3, pp.589-598, 2015.

[10] S. S. Kara, S. Onut, "A two-stage stochastic and robust programming approach to strategic planning of a reverse supply network: the case of paper recycling," Expert Systems with Applications, vol.37, no.9, pp.6129-6137, 2010.

[11] O. Listeş, R. Dekker, "A stochastic approach to a case study for product recovery network design,” European Journal of Operational Research, vol.160, no.1, pp.268-287, 2005.

[12] H. Ijuin, T, Yamada, Y. Kinoshita, A. Ishigaki, M. Inoue, "Design of reverse supply chain network with material recovery analysis," Journal of The Society of Plant Engineers Japan, vol.28, no.4, pp.147-159 2017 (in Japanese). 
[13] H. Ijuin, T, Yamada, A. Ishigaki, M. Inoue, "Modeling and analysis of reverse supply chain network with end-of-life product status for costs and recycling rate," Northeast Decision Science Institute 2016 Annual Meeting, pp.120-125, Alexandria, VA,USA, March, 2016.

[14] H. Ijuin, T, Yamada, A. Ishigaki, M. Inoue, "Reverse supply chain flows with material recovery constraint," The 12th Biennial International Conference on Ecobalance 2016, Kyoto, Japan, October 2016.

[15] F.S. Hillier, G.J. Lieberman, Introduction to Operations Research 8th Edition, McGrawHill, New York, NY, USA, 2005.

[16] Hitachi, Ltd., EcoAssist, http://www.ecoassist.com/HTML_n/option/rem/rem_tr/ppframe. htm (Accessed on Feb. 12, 2019), (in Japanese).

[17] T. Akahori, Y. Matsuno, Y. Adachi, N. Yamamoto, Y. Hamatsuka, T. Nishi, “Application of REM (Recyclability Evaluation Method) to home electric appliances: evaluation of recycling rates and costs," Journal of Japan Society of Waste Management Experts, vol.19, no.1, pp.4450, 2008 (in Japanese).

[18] K. Igarashi, T. Yamada, M. Inoue, "2-stage optimal design and analysis for disassembly system with environmental and economic parts selection using the recyclability evaluation method," International Journal of Industrial Engineering and Management Systems, vol.13 no.1, pp.52-66, 2014. 